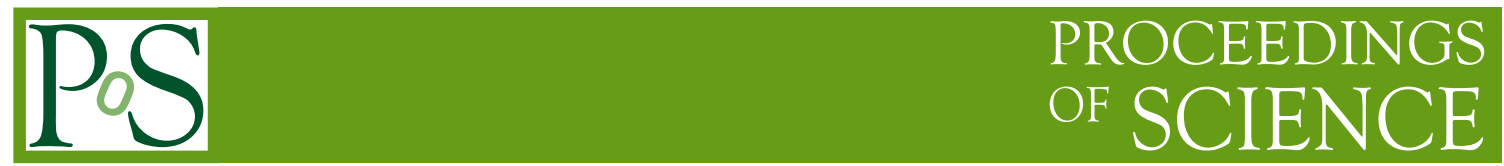

New forms of potential for string axion inflation

\title{
Tatsuo Kobayashi*i
}

Department of Physics, Hokkaido University, Sapporo 060-0810, Japan

E-mail: kobayashi@particle.sci.hokudai.ac.jp

\begin{abstract}
We study two new types of axion inflation potentials. One is induced by the gaugino condensation with one-loop threshold corrections. The Dedekind eta function appears in the axion inflation potential. The other is induced by quantum corrections on the period vector on the Calabi-Yau manifold. That induces the inflation potential, which is a mixture of polynomial functions and sinusoidal functions of the axion. That leads to unique behavior. In the latter potential, both the large-field inflation scenario and the small-field inflation scenario can be realized depending on potential parameters.
\end{abstract}

The 11th International Workshop Dark Side of the Universe 2015

14-18 December 2015

Kyoto, Japan

* Speaker.

${ }^{\dagger}$ A footnote may follow. 


\section{Introduction}

There are many moduli in superstring theory on the 6D compact space. Their vacuum expectation values (VEVs) represent the size and shape of the compact space. Thus, moduli provide us with unique characters of superstring theory on the compact space. Indeed, moduli fields play an important role in particle phenomenology and cosmology. The gauge couplings and Yukawa couplings as well as other couplings are determined by VEVs of moduli.

Moduli fields have perturbatively flat potential. In particular, the imaginary parts of moduli, i.e. axions, have a shift symmetry leading to a flat potential. Thus, axions as well as moduli would be a good candidate for the inflaton. Non-perturbative effects would break the shift symmetry into discrete one, and induce the potential of the axion. For example, such effects would generate the following potential of the axion $a$,

$$
V=C(1-\cos (a / f))
$$

where $f$ is the decay constant. That is nothing but the potential for the so-called natural inflation [1]. We need super-Planckian decay constant, $f \gtrsim 5 M_{P}$, where $M_{P}$ denotes the reduce Planck, in order to realize sufficient e-fold. Hereafter, we use the unit that $M_{P}=1$. Within the framework of superstring theory, the typical decay constant would be sub-Planckian, $f<M_{P}$ [2]. However, the super-Planckian decay constant can be obtained effectively by alignment of more than two axions with sub-Planckian decay constants [3]. Also one-loop effects would be helpful to enlarge the decay constant $[4,5]$.

Another type of the axion inflation potential is as follows,

$$
V=C a^{r},
$$

with $r=2,1,2 / 3$, etc. This potential is derived within the axion monodromy inflation [6], ${ }^{1}$ where the value $r$ depends on the setup of D-branes and fluxes.

Here, we discuss the possibilities for other types of axion inflation potentials. We study two new types of the axion inflation potentials. One type of axion potentials is generated through the gaugino condensation in the hidden sector. We consider the scenario that our inflaton-axion does not appear in the gauge kinetic function at tree level, but it appears in one-loop threshold corrections on the gauge kinetic function, which is written by the Dedekind eta function. Thus, the Dedekind eta function of the inflaton-axion appears in the inflation potential [5]. The other type of the axion potential is induced by quantum corrections on period vectors of Calabi-Yau manifolds [8], while the corresponding axion is massless at tree level. Such quantum corrections lead to a very unique axion potential, and its resultant potential form is a kind of mixture of polynomial functions and sinusoidal functions of the axion. Both of the potential forms look novel, and would lead to interesting aspects.

This paper is organized as follows. In section 2, we study the axion potential generated through the hidden-sector gaugino condensation with one-loop threshold corrections. The Dedekind eta function of the inflaton-axion appears in the inflation potential. In section 3, we study the axion potential, which is induced by quantum corrections on the period vectors of Calabi-Yau manifolds.

\footnotetext{
${ }^{1}$ See also [7].
} 
In our model, there is an axion, which is massless at the tree-level, but quantum corrections generate its potential, which is a kind of mixture of polynomial functions and sinusoidal functions of the axion. In section 4 , we give a comment on reheating process after inflation. In our scenario, the inflaton does not appear in the gauge kinetic function at tree-level, but may appear in one-loop gauge kinetic function and/or Yukawa couplings. That may lead rather low reheating temperature. Section 5 is conclusion and discussion.

\section{Threshold corrections}

In superstring theory, non-perturbative effects such as D-brane instanton effects and gaugino condensation in the hidden sector would induce the following form of superpotential of the modulus, $T=t+i a$ :

$$
W=A e^{-c T}+\cdots .
$$

Then, this superpotential would lead to the scalar potential of the axion $a$,

$$
V=(1-\cos (a / f))+\cdots,
$$

where $f=1 / c$. This is nothing but the potential for the natural inflation.

One of possible non-perturbative effects to generate the above superpotential term is the gaugino condensation by strong coupling dynamics in the hidden sector. We assume that this hidden sector is pure supersymmetric Yang-Mills theory without matter fields, that is, the theory with the gaugino fields and gauge bosons. Suppose that the gauge kinetic function of this hidden sector is written by

$$
f_{\text {hidden }}=T \text {. }
$$

That is, the gauge coupling $g$ is written by $1 / g^{2}=\operatorname{Re}(T)$. This hidden sector is asymptotically free and gaugino fields would condensate at the dynamical scale $\Lambda_{d}$. The dynamical scale is evaluated as $\Lambda_{d}=M_{P} e^{-8 \pi^{2} / b g^{2}}$, where $b$ denotes the one-loop beta function coefficient of the hidden gauge sector. For example, we obtain $b=3 N$ for the $S U(N)$ pure supersymmetric Yang-Mills theory. Then, non-perturbative superpotential term (2.1) of the modulus $T$ would appear,

$$
W=A e^{-8 \pi^{2} T / N},
$$

where $A \sim M_{P}^{3}$. In this case, we obtain the decay constant,

$$
f=\frac{1}{c}=\frac{N}{8 \pi^{2}} .
$$

We would need a large gauge group, e.g. $N=\mathscr{O}(100)$ in order to realize $f \sim 5 M_{p}$.

In general, the gauge kinetic function has one-loop corrections, where another modulus $M$ appears,

$$
f_{\text {hidden }}=T+\frac{\Delta(M)}{16 \pi^{2}} .
$$

Again, the gaugino condensation would induce non-pertrubative superpotential of $M$. In this case, the axion of $M$ could have larger decay constant, because the inverse of the loop factor $16 \pi^{2}$ enlarges the decay constant $[4,5]$. 
Indeed, one-loop threshold corrections were calculated in several string models. For example, in heterotic string theory on orbifolds, the gauge kinetic function at one-loop level is obtained by [9]

$$
f_{\text {hidden }}=S+\frac{\Delta}{16 \pi^{2}}, \quad \Delta=4 b_{N=2} \ln \eta(T),
$$

where $S$ and $T$ are the dilaton and Kähler modulus, $b_{N=2}$ denotes one-loop beta function coefficient due to the $N=2$ sector, and $\eta(T)$ is the Dedekind eta function,

$$
\eta(T)=e^{-\pi T / 12} \prod_{m}\left(1-e^{-2 \pi m T}\right) .
$$

In addition to the Kähler modulus, the complex moduli may also appear in threshold corrections $\Delta$ of certain models.

Similarly, in type IIA intersecting D-brane models, the gauge kinetic function is otained by [10],

$$
f_{\text {hidden }}=a S+c U+\frac{\Delta}{16 \pi^{2}}, \quad \Delta=4 b_{N=2} \ln \eta(T),
$$

where $a$ and $c$ are some constants determined by D-brane configuration, and $U$ denotes the complex structure modulus. Their T-dual corresponds to the type IIB D-brane models, where the gauge kinetic function would be written by

$$
f_{\text {hidden }}=a S+c T+\frac{\Delta}{16 \pi^{2}}, \quad \Delta=4 b_{N=2} \ln \eta(U) .
$$

Similarly, other types of string models would also have one-loop corrections in the gauge kinetic functions.

Now, let us study the type IIB theory for concreteness. We consider the hidden sector with the above gauge kinetic function (2.10). Then, the gaugino condensation in the hidden sector would induce non-perturbative superpotential,

$$
W=w_{0}+A(S, T) \eta(U)^{-3 b_{N=2} / b \pi}
$$

where $w_{0}$ is constant and $A(S, T)$ is a function of $S$ and $T$. We assume that all of moduli except $a=\operatorname{Im}(U)$ are stabilized. Thus, by substituting the superpotential into the scalar potential,

$$
V=e^{K}\left(\left|D_{U} W\right|^{2}-3|W|^{2}\right),
$$

where $K=-\ln (U+\bar{U})$ and $D_{U} W=K_{U} W+W_{U}$, we derive a new type of scalar potential of the axion, which is obtained through the Dedekind eta function. Also, in order to realize slightly positive vacuum energy, we must add the uplifting term by explicit supersymmetry (SUSY) breaking effects [11] or spontaneous F-term SUSY breaking effects [12, 13].

When $\operatorname{Re}(U)$ is large, the Dedekind eta function can be approximated by

$$
\eta(U) \sim e^{-\pi U / 12}
$$


In such a case, our potential reduces the potential for the natural inflation (1.1) with the decay constant,

$$
f=\frac{6 \operatorname{Re}(U) N}{b_{N=2} \pi},
$$

for the $S U(N)$ hidden gauge group. In this model, we can realize a large decay constant such as $f \gtrsim 5 M_{p}$ for smaller hidden gauge group because the inverse of the loop factor, $16 \pi^{2}$, enlarges the decay constant. For example, when $\operatorname{Re}(U)=1.3, b_{N=2}=1$ and $N=4$, we realize $f \sim 10$, and we obtain the observables,

$$
N_{e}=50, \quad n_{s}=0.96, \quad r=0.11, \quad \alpha_{S}=-10^{-3},
$$

where $N_{e}$ is e-fold number, $n_{s}$ is the spectral index, $r$ is the tensor-to-scalar ratio, and $\alpha_{S}$ is running of the spectral index.

For smaller value of $\operatorname{Re}(U)$, more corrctions in the Dedekind eta function become important, i.e.

$$
\eta(U)=e^{\pi} U / 12\left(1-e^{-2 \pi U}\right)+\cdots .
$$

That would lead to the scalar potential,

$$
V=\Lambda(1-\cos (c a))+\Lambda_{2} \cos \left(c_{2} a\right)+\cdots
$$

with $c=b_{N=2 \pi} / 6 \operatorname{Re}(U) N$ for the hidden $S U(N)$. The second term is modulation and their coefficients are related with ones in the first terms as

$$
\begin{aligned}
\Lambda_{2} & =\Lambda \frac{2 b_{N=2}}{N} e^{-(c+2 \pi / R e(U))}, \\
c_{2} & =\frac{2 \pi+c}{\operatorname{Re}(U)} .
\end{aligned}
$$

The potential itself is not significantly different between with and without the correction term, but its derivatives drastically change by the correction term. Hence, the modulation term is quite important and observables change depending on the potential parameters. See for detail of numerical analysis Ref. [5].

Also, in Ref. [15], another inflation potential including the Dedekind eta function and its derivative was studied. In the model, the modular invariance is required and quite different aspects appear. (See in detail Ref. [15].)

\section{Quantum corrected period vector}

Here, we study another new type of axion inflation potential. In type IIB superstring theory, non-vanshing three-form fluxes induce a superpotential term for complex structure moduli and dilaton [14],

$$
\begin{aligned}
W_{G V W} & =\int\left(F_{3}-i S H_{3}\right) \wedge \Omega(U), \\
& =\sum_{\alpha}\left(N_{F}-i S N_{F}\right)^{\alpha} \Pi_{\alpha}(U),
\end{aligned}
$$


where $F_{3}$ and $H_{3}$ are Ramond-Ramond and Neveu-Schwarz three-form fluxes, respectively, and $\Omega(U)$ denotes the holomorphic three form of the Calabi-Yau manifold. Here, $N_{F}$ and $H_{F}$ are integer and correspond to $F_{3}$ and $H_{3}$, and $\alpha$ runs from 1 to $2 h^{1,2}+2$, where $h^{1,2}$ is the number of complex structure moduli, i.e., the hodge number of the Calabi-Yau manifold. In addition, $\Pi_{\alpha}$ denotes the period vector, which is polynomial functions of $U$ at tree level. Some or all of complex structure moduli and dilaton can be stabilized through this superpotential by introducing proper three-form fluxes.

Quantum corrections on the period vector were computed [16] (see also [17, 18]). The period vector,

$$
\Pi_{\alpha}=\left(\begin{array}{c}
1 \\
U^{i} \\
2 F-U^{i} \partial_{i} F \\
\partial_{i} F
\end{array}\right)
$$

is written by the prepotential $F$, and the quantum corrected prepotential is obtained as

$$
\begin{aligned}
F= & -\frac{1}{3 !} \kappa_{i j k} U^{i} U^{j} U^{k}-\frac{1}{2} \kappa_{i j} U^{i} U^{j}+\kappa_{i} U^{i}+\frac{1}{2} \kappa_{0} \\
& -\frac{1}{(2 \pi i)^{3}} \sum_{\beta} n_{\beta} \operatorname{Li}_{3}\left(e^{2 \pi i d_{i} U^{i}}\right),
\end{aligned}
$$

where $\mathrm{Li}_{3}(z)$ is the polylogarithm function, i.e.

$$
\operatorname{Li}_{s}(z)=\sum_{n=1} \frac{z^{n}}{n^{s}}
$$

and $n_{\beta}$ is the genus zero Gromov-Witten invariants (world-sheet instanton) labeled by $\beta=d_{i} \beta_{i}$ with $d_{i}$ and $\beta_{i}$ being the integer and the elements in cohomology of the mirror Calabi-Yau monifold $\tilde{M}_{\mathrm{CY}}$. The parameters, $\kappa_{i j k}, \kappa_{i j}, \kappa_{i}$ and $\kappa_{0}$ are given by

$$
\begin{gathered}
\kappa_{i j k}=\int_{\tilde{M}_{\mathrm{CY}}} J_{i} \wedge J_{i} \wedge J_{k}, \quad \kappa_{i j}=-\frac{1}{2} \int_{\tilde{M}_{\mathrm{CY}}} J_{i} \wedge J_{i} \wedge J_{i}, \\
\kappa_{i}=\frac{1}{43 !} \int c_{2}\left(\tilde{M}_{\mathrm{CY}}\right) \wedge J_{i}, \quad \kappa_{0}=\frac{\zeta(3)}{(2 \pi i)^{3}} \chi\left(\tilde{M}_{\mathrm{CY}}\right),
\end{gathered}
$$

where $\zeta(3) \simeq 1.2$ and $\chi\left(\tilde{M}_{\mathrm{CY}}\right)$ denotes the Euler character. The Kähler potential $K$ for the complex structure moduli is also written by use of the the period vector and prepotential,

$$
e^{-K(U, \bar{U})}=i \Pi^{\dagger} \cdot \Sigma \cdot \Pi=\left(2(F-\bar{F})-\left(U^{i} \bar{U}^{i}\right)\left(F_{i}+\bar{F}_{i}\right)\right),
$$

where $\Sigma$ is the symplectic matrix,

$$
\Sigma=\left(\begin{array}{cc}
0 & \mathbf{1} \\
-1 & 0
\end{array}\right)
$$


More explicitly, the Kähler potential is written by

$$
\begin{aligned}
e^{-K(U, \bar{U})}= & f_{0}(U, \bar{U}) \\
& +\frac{2}{(2 \pi)^{2}} \sum_{\beta} \sum_{n=1}^{\infty} d_{i} n_{\beta}\left(U^{i}+\bar{U}^{i}\right) \frac{1}{n^{2}} \cos \left(-i \pi n \sum_{j} d_{j}\left(U^{i}-\bar{U}^{j}\right)\right) e^{-\pi n \sum_{k} d_{k}\left(U^{k}+\bar{U}^{k}\right)} \\
& +\frac{4}{(2 \pi)^{3}} \sum_{\beta} \sum_{n=1}^{\infty} n_{\beta} \frac{1}{n^{3}} \cos \left(-i \pi n \sum_{j} d_{j}\left(U^{i}-\bar{U}^{j}\right)\right) e^{-\pi n \sum_{k} d_{k}\left(U^{k}+\bar{U}^{k}\right)},
\end{aligned}
$$

with

$$
f_{0}(U, \bar{U})=\frac{1}{6} \sum_{i j k} \kappa_{i j k}\left(U^{i}+\bar{U}^{i}\right)\left(U^{j}+\bar{U}^{j}\right)\left(U^{k}+\bar{U}^{k}\right)-\frac{\zeta(3)}{4 \pi^{3}} \chi\left(\tilde{M}_{\mathrm{CY}}\right)
$$

Here and hereafter we write $U^{i}$ as $i U^{i}$.

Now, we introduce the three-form fluxes such that two complex structure moduli, $U_{1}$ and $U_{2}$ appear in the superpotential as

$$
W(S, U)=g_{0}(z)+g_{1}(z)\left(U_{2}+N U_{1}\right)
$$

at tree level, where $z$ denote the other complex structure moduli and the dilaton. Their Kähler potential is also written

$$
K=-\ln \left[f_{0}\left(\operatorname{Re}(z), \operatorname{Re}\left(U_{1}\right), \operatorname{Re}\left(U_{2}\right)\right)\right] .
$$

at tree level. Then, this superpotential and Kähler potential include only the linear combination of the imaginary parts, $\operatorname{Im}\left(U_{2}\right)+N \operatorname{Im}\left(U_{1}\right)$. Thus, one axionic mode remains massless at this level. Following Ref. [19], we redefine the field basis,

$$
\begin{aligned}
& \Psi=U_{2}+N U_{1}, \\
& \Phi=U_{2} .
\end{aligned}
$$

Their Kähler potential and superpotential are written by

$$
\begin{aligned}
K & =-\ln \left[f_{0}(\operatorname{Re}(z), \operatorname{Re}(\Psi-\Phi) / N, \operatorname{Re}(\Phi))\right], \\
W & =g_{0}(z)+g_{1}(z) \Psi .
\end{aligned}
$$

The complex modulus $\Psi$ can be stabilized at $D_{\Psi} W=0$. Also $\operatorname{Re}(\Phi)$ can be stabilized at $K_{\Phi}=0$ with non-vanishing superpotential VEV. However, the axionic mode $\operatorname{Im}(\Phi)$ does not appear in the Kähler potential and the superpotential, and it remains masslees at this level.

Now, let us study the quantum corrections, which include the following corrections on the Kähler potential and the superpotential,

$$
\begin{aligned}
& \Delta K \simeq-\frac{1}{\left\langle f_{0}\right\rangle} \sum_{i=1}^{2} f_{1}^{(i)}\left(\frac{2}{\pi}+U_{i}+\bar{U}_{i}\right) \cos \left(-i \pi\left(U_{i}-\bar{U}_{i}\right)\right) e^{-\pi\left(U_{i}+\bar{U}_{i}\right)}, \\
& \Delta W \simeq \sum_{i=1}^{2}\left(g_{2}^{(i)}+g_{3}^{(i)} U_{i}\right) e^{-2 \pi U_{i}}
\end{aligned}
$$


where $f_{1}^{(i)}$ and $g_{2,3}^{(i)}$ depend on VEVs of heavy moduli. We omit the exponential term for $U_{2}$ under the condition,

$$
e^{-2 \pi\left\langle\operatorname{Re}\left(U_{2}\right)\right\rangle} \ll e^{-2 \pi\left\langle\operatorname{Re}\left(U_{1}\right)\right\rangle} \ll 1 .
$$

Then, the Kähler potential and the superpotential reduce to

$$
\begin{aligned}
\Delta K & \left.\simeq-\frac{f_{1}^{(1)}}{\left\langle f_{0}\right\rangle}\left(\frac{2}{\pi}+\frac{\Psi+\bar{\Psi}-\Phi-\bar{\Phi}}{N}\right) \cos \left(-i \pi \frac{\Psi-\bar{\Psi}-\Phi+\bar{\Phi}}{N}\right)\right) e^{-\pi \frac{\Psi+\bar{\Psi}-\Phi-\bar{\Phi}}{N}}, \\
\Delta W & \simeq\left(g_{2}^{(1)}+\frac{g_{3}^{(1)}}{N}(\Psi-\Phi)\right) e^{-2 \pi \frac{\Psi-\Phi}{N}},
\end{aligned}
$$

on the field basis $(\Psi, \Phi)$. Obviously, these correction terms include $\operatorname{Im}(\Phi)$, and its axion potential is induced at quantum level.

We have assumed that the heavy complex moduli and the dilation are stabilized at the SUSY minimum. In addition, here we assume that the Kähler moduli are stabilized in the Kachru-KalloshLinde-Trivedi (KKLT) scenario [11] or the large volume scenario [20], their masses are sufficiently heavier than the light axion, $\operatorname{Im}(\Phi)$, and the stabilization mechanism of the Kähler modluli is independent of the light axion.

In the KKLT scenario, we can derive the following axion potential,

$$
V \simeq \Lambda_{1}\left(1-\cos \frac{\phi}{M_{1}}\right)+\lambda_{2} \phi \sin \frac{\phi}{M_{1}}
$$

in the limit $\left\langle\operatorname{Re}\left(U_{1}\right)\right\rangle \gg 1$, where $\phi=k_{1}\left(\langle\operatorname{Im}(\Psi)-\operatorname{Im}(\Phi))\right.$ with $k_{1}$ being the normalization factor of $\mathscr{O}\left(\left\langle\operatorname{Re}\left(U_{2}\right)\right\rangle\right)$. (See for derivation of the potential Ref. [8].) Here, we obtain $\Lambda_{1} \sim \Lambda_{2}$ and $M_{1}=N K_{1} / 2 \pi$. Thus, we can realize a large decay constant for larger flux $N$. The first term is the term for the natural inflation. Thus, this potential looks slightly similar to the natural inflation with a small modulation. However, the second term is not small correction in our model. At any rate, this is a new type of the inflation potential, which is derived from superstring theory. Note that we set a generic flux configuration, while the limited flux configuration was set in Ref. [19] to realize the simple natural inflation with a large decay constant.

Also, in the KKLT scenario, we can derive the following axion potential,

$$
V \simeq \Lambda_{3} \phi^{2}
$$

This potential is nothing but the quadratic chaotic inflation potential, which is disfavored by the recent result of Planck [21],

$$
\begin{aligned}
P_{\xi} & =2.20 \pm 0.10 \times 10^{-9}, \\
m_{s} & =0.9655 \pm 0.0062, \\
r & <0.11
\end{aligned}
$$

On the other hand, in the large volume scenario, we can derive the following axion potential,

$$
V \simeq \Lambda_{4} \phi^{2}+\Lambda_{5}\left(1-\cos \frac{\phi}{M_{3}}\right)+\lambda_{6} \phi \sin \frac{\phi}{M_{3}},
$$


where $\Lambda_{4} \sim \Lambda_{5} \sim \Lambda_{6}$ and $M_{3}=N K_{3} / 2 \pi$, and $\phi=k_{3}\left(\langle\operatorname{Im}(\Psi)-\operatorname{Im}(\Phi))\right.$ with $k_{3}$ being the normalization factor of $\mathscr{O}\left(\left\langle\operatorname{Re}\left(U_{2}\right)\right\rangle\right)$. (See for derivation of the potential Ref. [8].) In a certain parameter region, we obtian a hierarchy such as $\Lambda_{5,6} \gg \Lambda_{4}$. Again, this is a new type of the axion inflation potential, which is derived from superstring theory.

Finally, we show some examples of numerical analysis. Table 1 shows the observables in the axion inflation potential (3.18) with some parameters. All of these values are consistent with the Planck data. This axion inflation corresponds to the large-field inflation scenario.

\begin{tabular}{|cccccc|}
\hline$M_{1}$ & $\Lambda_{2} / \Lambda_{1}$ & $N_{e}$ & $n_{s}$ & $r$ & $d n_{s} / d \ln k$ \\
\hline 8 & 5 & 50 & 0.960 & 0.040 & -0.0005 \\
8 & 5 & 60 & 0.964 & 0.030 & -0.0003 \\
10 & 5 & 50 & 0.964 & 0.055 & -0.0006 \\
10 & 5 & 60 & 0.969 & 0.041 & -0.0004 \\
12 & 5 & 50 & 0.966 & 0.063 & -0.0006 \\
12 & 5 & 60 & 0.971 & 0.049 & -0.0004 \\
15 & 5 & 50 & 0.968 & 0.070 & -0.0006 \\
15 & 5 & 60 & 0.973 & 0.056 & -0.0004 \\
\hline
\end{tabular}

Table 1: Observables in the axion potential (3.18).

Similarly, Table 2 shows the observables in the axion inflation potential (3.21) with some parameters. All of these values are consistent with the Planck data. Most of these parameters correspond to the large-field inflation scenario. However, the fourth and fifth examples lead to $r<0.01$, and these correspond to the small-field inflation scenario. In these cases, there appear plateaus in the inflation potential. Thus, when the initial values of the inflaton starts around such a flat plateau, the tensor-to-scalar ratio is small and the movement in the field space during the inflation is rather small. We do not fine-tune the parameters to realize the small-field inflation. If we fine-tune the parameters, we could realize smaller $r$. At any rate, it is quite interesting that one inflation potential can lead to both the large-field inflation scenario and the small-field inflation scenario depending on parameter values. We would study its detail in further work.

\begin{tabular}{|ccccccc|}
\hline$M_{1}$ & $\Lambda_{4} / \Lambda_{6}$ & $\Lambda_{5} / \Lambda_{6}$ & $N_{e}$ & $n_{s}$ & $r$ & $d n_{s} / d \ln k$ \\
\hline 5 & 1 & 5 & 60 & 0.969 & 0.050 & -0.0007 \\
5 & 1 & 5 & 55 & 0.965 & 0.060 & -0.0008 \\
5 & 1 & 5 & 50 & 0.962 & 0.070 & -0.0009 \\
3 & 1 & 5 & 60 & 0.970 & 0.0008 & -0.0009 \\
3 & 1 & 5 & 55 & 0.964 & 0.0097 & -0.0009 \\
3 & 1 & 5 & 50 & 0.956 & 0.012 & -0.0009 \\
5 & $1 / 5$ & 1 & 60 & 0.968 & 0.050 & -0.0007 \\
5 & $1 / 5$ & 1 & 55 & 0.965 & 0.060 & -0.0008 \\
\hline
\end{tabular}

Table 2: Observables in the axion potential (3.21). 


\section{Comment on reheating}

In the inflation model of section 2, the axion field appears not in tree-level gauge kinetic function, but in one-loop one. Also, in the inflation model of section 3, the inflaton is the axion of the complex structure moduli, which would appear not in the tree-level gauge kinetic function, but in the one-loop gauge kinetic function. Thus, the inflaton fields in these models have suppressed couplings with the gauge sector, and would have a characteristic feature in thermal history after inflation. Here, we give a brief comment on reheating.

As mentioned above, the inflaton field in our models appears through the moduli-dependent one-loop corrections,

$$
-\frac{1}{4 g_{a}^{2}} F_{\mu \nu}^{a} F^{a \mu \nu}-\frac{1}{4} \frac{\Delta(\Phi)}{16 \pi^{2}} F_{\mu \nu}^{a} F^{a \mu \nu},
$$

where $a=1,2,3$ correspond to the gauge groups of the standard model, $U(1)_{Y}, S U(2)$ and $S U(3)$, respectively. Through these couplings, the inflaton decays into the gauge bosons $g^{(a)}$, and its decay width is estimated as

$$
\begin{aligned}
\Gamma_{\phi} & =\sum_{a=1}^{3} \Gamma\left(\phi \rightarrow g^{(a)}+g^{(a)}\right) \\
& =\sum_{a=1}^{3} \frac{N_{G}^{a}}{128 \pi}\left(\frac{\partial_{\phi}(\Delta(\Phi)) g_{a}^{2}}{16 \pi^{2} d}\right)^{2} \frac{m_{\phi}^{3}}{M_{P}^{2}} \\
& \simeq 5.8 \times 10^{-5}\left(\frac{m_{\phi}}{10^{13} \mathrm{GeV}}\right)^{3} \mathrm{GeV},
\end{aligned}
$$

where $\sum_{a=1}^{3} N_{G}^{a}=12, d=\mathscr{O}\left(\sqrt{K}_{\Phi \bar{\Phi}}\right)=\mathscr{O}(1), g_{a}^{2} \simeq 0.53$, and $m_{\phi}$ denotes the inflation mass. Now, we assume that $\Delta(\Phi)=\Phi$. Then, when the above decay is dominant to the total decay width of the inflaton decay, the reheating temperature is estimated by $\Gamma_{\phi} \simeq H\left(T_{R}\right)$. That is, the reheating temperature is evaluated by

$$
\begin{aligned}
T_{R} & =\left(\frac{\pi^{2} g_{*}}{90}\right)^{-1 / 4} \sqrt{\Gamma_{\phi} M_{P}} \\
& \simeq 6.4 \times 10^{6}\left(\frac{m_{\phi}}{10^{13} \mathrm{GeV}}\right)^{3 / 2} \mathrm{GeV},
\end{aligned}
$$

where $g_{*}=106.75$ is the effective degrees of freedom of the radiation at the reheating in the standard model. The reheating temperature would be rather low.

In type IIB superstring theory, the complex moduli would appear in Yukawa couplings and other couplings with matter fields in the superpotential. (See for concrete computations, e.g. [22].) Then, the inflaton could decay into matter fields through such couplings. When such decays are dominatn, a similar computation leads to the following reheating temperature,

$$
T_{R} \simeq 8.8 \times 10^{7}\left(\partial_{\Phi} Y_{i j k}\right)\left(\frac{m_{\phi}}{10^{13} \mathrm{GeV}}\right)^{3 / 2} \mathrm{GeV},
$$

where $\partial_{\Phi} Y_{i j k}$ denotes the first derivative of moduli-dependent Yukawa couplings $Y_{i j k}$. The estimated reheating temperature may also be rather low, although it depends on $\partial_{\Phi} Y_{i j k}$. 


\section{Conclusion}

We have studied two new types of the axion inflation potentials. One is induced by the hidden sector gaugino condensation with one-loop threshold corrections. The threshold correction is given by the Dedekind eta function and the axion appears in the inflation potential through the Dedekind eta function. When the real part VEV of the inflaton modulus is large, this potential reduces to the potential for the natural inflation. However, when the real part VEV becomes smaller, the modulation becomes important. The inverse of the one-loop factor, $16 \pi^{2}$, is helpful to enlarge the decay constant.

The other type is induced by quantum corrected period vector. The axion potential is completely flat at tree level. However, the quantum corrections induce non-trivial potential for the axion, and its form is a mixture of polynomial functions and sinusoidal functions. In some parameter region, that leads to the large-field inflation scenario, but other region leads to the small-field inflation scenario. Although we showed some examples of the small-field inflation scenario without fine-tuning, we can realize much smaller tensor-to-scalar ratio by fine-tuning. At any rate, it is quite interesting that one inflation model can lead to both the large-field inflation scenario and the small-field inflation scenario depending on parameter values. We would study more detail in further work [23].

The inflaton in our models would couple with the gauge bosons through one-loop corrections and/or with matter fields through Yukawa couplings. Then, we can estimate the inflation decay. The reheating temperature may be rather low because those couplings are suppressed.

\section{Acknowledgments}

The author would like to thank H.Abe and A.Oikawa and H.Otsuka for collaborations, on which this talk is based. T. K. was supported in part by the Grant-in-Aid for Scientific Research No. 25400252 and No. 26247042 from the Ministry of Education, Culture, Sports, Science and Technology (MEXT) in Japan.

\section{References}

[1] K. Freese, J. A. Frieman and A. V. Olinto, Phys. Rev. Lett. 65 (1990) 3233.

[2] P. Svrcek and E. Witten, JHEP 0606, 051 (2006) doi:10.1088/1126-6708/2006/06/051 [hep-th/0605206].

[3] J. E. Kim, H. P. Nilles and M. Peloso, JCAP 0501, 005 (2005) doi:10.1088/1475-7516/2005/01/005 [hep-ph/0409138].

[4] H. Abe, T. Kobayashi and H. Otsuka, PTEP 2015, no. 6, 063E02 [arXiv:1409.8436 [hep-th]].

[5] H. Abe, T. Kobayashi and H. Otsuka, JHEP 1504 (2015) 160 [arXiv:1411.4768 [hep-th]].

[6] E. Silverstein and A. Westphal, Phys. Rev. D 78 (2008) 106003 [arXiv:0803.3085 [hep-th]].

L. McAllister, E. Silverstein and A. Westphal, Phys. Rev. D 82, 046003 (2010) doi:10.1103/PhysRevD.82.046003 [arXiv:0808.0706 [hep-th]];

R. Flauger, L. McAllister, E. Pajer, A. Westphal and G. Xu, JCAP 1006, 009 (2010) doi:10.1088/1475-7516/2010/06/009 [arXiv:0907.2916 [hep-th]]; 
X. Dong, B. Horn, E. Silverstein and A. Westphal, Phys. Rev. D 84, 026011 (2011) doi:10.1103/PhysRevD.84.026011 [arXiv:1011.4521 [hep-th]].

[7] T. Kobayashi, O. Seto and Y. Yamaguchi, PTEP 2014, no. 10, 103E01 (2014) doi:10.1093/ptep/ptu145 [arXiv:1404.5518 [hep-ph]];

T. Higaki, T. Kobayashi, O. Seto and Y. Yamaguchi, JCAP 1410, no. 10, 025 (2014) doi:10.1088/1475-7516/2014/10/025 [arXiv:1405.0775 [hep-ph]].

[8] T. Kobayashi, A. Oikawa and H. Otsuka, arXiv:1510.08768 [hep-ph].

[9] L. J. Dixon, V. Kaplunovsky and J. Louis, Nucl. Phys. B 355, 649 (1991).

[10] D. Lust and S. Stieberger, Fortsch. Phys. 55, 427 (2007) doi:10.1002/prop.200310335 [hep-th/0302221].

[11] S. Kachru, R. Kallosh, A. D. Linde and S. P. Trivedi, Phys. Rev. D 68, 046005 (2003) doi:10.1103/PhysRevD.68.046005 [hep-th/0301240].

[12] O. Lebedev, H. P. Nilles and M. Ratz, Phys. Lett. B 636, 126 (2006) [hep-th/0603047].

[13] E. Dudas, C. Papineau and S. Pokorski, JHEP 0702 (2007) 028 [hep-th/0610297];

H. Abe, T. Higaki, T. Kobayashi and Y. Omura, Phys. Rev. D 75 (2007) 025019 [hep-th/0611024];

R. Kallosh and A. D. Linde, JHEP 0702 (2007) 002 [hep-th/0611183];

H. Abe, T. Higaki and T. Kobayashi, Phys. Rev. D 76 (2007) 105003 [arXiv:0707.2671 [hep-th]].

[14] S. Gukov, C. Vafa and E. Witten, Nucl. Phys. B 584, 69 (2000) Erratum: [Nucl. Phys. B 608, 477 (2001)] doi:10.1016/S0550-3213(00)00373-4 [hep-th/9906070].

[15] T. Kobayashi, D. Nitta and Y. Urakawa, arXiv:1604.02995 [hep-th].

[16] S. Hosono, A. Klemm, S. Theisen and S. T. Yau, Nucl. Phys. B 433, 501 (1995) doi:10.1016/0550-3213(94)00440-P [hep-th/9406055].

[17] K. Hori and C. Vafa, hep-th/0002222.

[18] Y. Honma and M. Manabe, JHEP 1305, 102 (2013) doi:10.1007/JHEP05(2013)102 [arXiv:1302.3760 [hep-th]].

[19] A. Hebecker, P. Mangat, F. Rompineve and L. T. Witkowski, Phys. Lett. B 748, 455 (2015) doi:10.1016/j.physletb.2015.07.026 [arXiv:1503.07912 [hep-th]].

[20] V. Balasubramanian, P. Berglund, J. P. Conlon and F. Quevedo, JHEP 0503, 007 (2005) doi:10.1088/1126-6708/2005/03/007 [hep-th/0502058].

[21] P. A. R. Ade et al. [Planck Collaboration], arXiv:1502.02114 [astro-ph.CO].

[22] D. Cremades, L. E. Ibanez and F. Marchesano, JHEP 0405, 079 (2004) [hep-th/0404229];

H. Abe, K. S. Choi, T. Kobayashi and H. Ohki, JHEP 0906, 080 (2009) [arXiv:0903.3800 [hep-th]].

[23] K. Kadota, T. Kobayashi, A. Oikawa, N. Omoto, H. Otsuka and T.H. Tatsuishi, in preparation. 